\title{
Open
}

\section{Bile retinoids imprint intestinal CD103+ dendritic cells with the ability to generate gut-tropic T cells}

\author{
E Jaensson-Gyllenbäck ${ }^{1,4}$, K Kotarsky $^{1,4}$, F Zapata ${ }^{1}$, EK Persson ${ }^{1}$, TE Gundersen ${ }^{2}$, R Blomhoff ${ }^{2,3}$ and \\ WW Agace ${ }^{1}$
}

Small intestinal lamina propria (SI-LP) $\mathrm{CD} 103^{+}$dendritic cells (DCs) are imprinted with an ability to metabolize vitamin $\mathrm{A}$ (retinol), a property underlying their enhanced capacity to induce the gut-homing receptors CC chemokine receptor- 9 and $\alpha 4 \beta 7$ on responding T cells. In this study, we demonstrate that imprinting of CD103+ DCs is itself critically dependent on vitamin A and occurs locally within the small intestine (SI). The major vitamin A metabolite retinoic acid (RA) induced retinol-metabolizing activity in DCs both in vitro and in vivo, suggesting a direct role for RA in this process. Consistent with this, SI-LP CD103+ DCs constitutively received RA signals in vivo at significantly higher levels than did colonic CD103+ DCs. Remarkably, SI CD103+ DCs remained imprinted in mice depleted of dietary but not of systemic retinol. We found that bile contained high levels of retinol, induced RA receptor-dependent retinol-metabolizing activity in bone marrow-derived DCs, and imprinted these cells with the ability to generate gut-tropic T cells. Taken together, these results suggest a novel and unexpected role for bile in SI-LP CD103+ DC imprinting.

\section{INTRODUCTION}

The intestinal immune system is continually exposed to foreign antigens in our diet and from the resident intestinal microflora, and must remain tolerant to these innocuous antigens while maintaining the ability to mount appropriate immune responses to mucosal pathogens.

Large numbers of macrophages and dendritic cells (DCs) are present within the intestinal lamina propria (LP) in the steady state and these cells, through their ability to regulate innate and adaptive immune responses, are essential in maintaining gut homeostasis (for reviews, see the studies by Coombes and Powrie $^{1}$ and Rescigno and Di Sabatino ${ }^{2}$ ).

$\mathrm{CD}_{103}{ }^{+}$, DCs that represent the dominant DC population in the murine small intestinal LP (SI-LP) and in the draining afferent lymph ${ }^{3-6}$ have a central role in initiating T-cell responses to luminal antigen in the draining mesenteric lymph node $(\mathrm{MLN}),{ }^{5,7,8}$ and have been implicated in mediating oral tolerance. ${ }^{8}$ Murine SI-LP and MLN CD $103^{+}$DCs in both humans and mice share several unique characteristics including an enhanced ability to induce the gut-homing receptors CC chemokine receptor (CCR) 9 and $\alpha 4 \beta 7$ on responding $\mathrm{T}$ cells and naive $\mathrm{CD}^{+}{ }^{+} \mathrm{T}$-cell conversion to inducible regulatory $\mathrm{T}$ cells, both of which are dependent on signaling events initiated by the vitamin A metabolite, retinoic acid (RA). 3,5,7,9-11

Vitamin A (retinol) is obtained through diet and is converted by small intestinal enterocytes to retinyl esters before being transported in chylomicrons through the lymph into the bloodstream. Circulating chylomicron remnants are taken up by stellate cells in the liver, which represents the major storage site of retinol in the body. Retinol, in complex with the retinol-binding protein (RBP)-4, is continually released from the liver into the circulation, and once in tissues, is converted into its active metabolite RA through a two-step enzymatic oxidation (for review, see the study by Blomhoff and Blomhoff ${ }^{12}$ ). Conversion of retinol to retinal is mediated by alcohol dehydrogenases, and retinal is irreversibly converted to RA by retinaldehyde dehydrogenases, the major isoform of which, retinaldehyde dehydrogenase-2, is encoded by aldh1a2. ${ }^{12}$ SI-LP and MLN CD $103^{+}$DCs display higher aldehyde dehydrogenase activity, aldh1a2 expression, and induce

${ }^{1}$ Department of Experimental Medical Sciences, Immunology Section, Lund University, Lund, Sweden. ${ }^{2}$ Vitas AS, Oslo Innovation Park, Oslo, Norway. ${ }^{3}$ Institute of Basic Medical Sciences, University of Oslo, Oslo, Norway. ${ }^{4}$ These authors contributed equally to this work. Correspondence: W Agace (William.Agace@med.lu.se) 
enhanced RA signaling in T cells compared with DCs outside the small intestine (SI). 5,7,13,14 Thus, the ability of SI CD $103^{+}$ DCs to efficiently induce gut-homing receptors and inducible regulatory T-cell differentiation, both properties of small intestinal immune responses, seems to be due, at least in part, to their enhanced ability to metabolize retinol.

We, along with others, have suggested that the SI environment modulates the phenotype and activity of SI-LP DCs or their precursors and imprints them with an enhanced ability to metabolize retinal. ${ }^{1,5,15-20}$ Although several factors induce aldh1a2 expression and/or aldehyde dehydrogenase activity in DCs in vitro including peroxisome proliferator-activated receptor- $\gamma$ agonists, ${ }^{21}$ toll-like receptor-2 ligands, ${ }^{22}$ interleukin (IL)- $4,{ }^{14,23}$ IL-13, ${ }^{14}$ granulocyte macrophage colony-stimulating factor, ${ }^{14}$ and RA itself,,${ }^{10,14}$ the relevance of these signals in steady-state imprinting of SI-LP CD103 ${ }^{+}$DCs in vivo remains

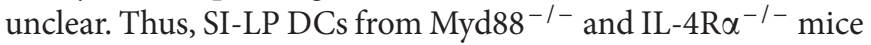
express normal levels of aldh $1 a 2$ and aldehyde dehydrogenase activity. ${ }^{13,24}$ Moreover, although total $\mathrm{CD} 11 \mathrm{c}^{+}$cells from the MLN of $\beta-\mathrm{C}^{-1-}$ (a common subunit in the granulocyte macrophage colony-stimulating factor, IL-3, and IL-5 receptor) mice show reduced aldehyde dehydrogenase activity, ${ }^{14}$ subsequent studies have shown a central role for granulocyte macrophage colony-stimulating factor in the development of $\mathrm{CD} 103^{+}$ DCs. ${ }^{24,25}$ Recently, CD11c ${ }^{+}$MLN cells in vitamin A-deficient (VAD) mice ${ }^{14}$ were shown to display reduced aldh1a2 expression and aldehyde dehydrogenase activity, implicating a possible role for retinol itself in DC imprinting. However, it remains unclear whether these effects were due to reduced local imprinting of CD103+ SI-LP DCs, and if so, how retinol contributes to the selective imprinting of cells in the SI.

In the current study, we identify a critical role for vitamin A in the local imprinting of SI-LP CD103 ${ }^{+}$DCs. Strikingly, such imprinting did not require dietary intake of retinol. Rather, we demonstrate that the liver releases high levels of retinol into the bile and that bile induces RA receptor (RAR)-dependent retinol-metabolizing activity in DCs and imprints them with the ability to generate gut-tropic $\mathrm{T}$ cells.

\section{RESULTS \\ Vitamin A is essential for the generation of gut-tropic $\mathrm{CD}^{+} \mathrm{T}$ cells in vivo}

Vitamin A is required for DC-induced generation of gut-tropic $\mathrm{T}$ cells in vitro. ${ }^{26,27}$ To determine the role of vitamin $\mathrm{A}$ in the generation of gut-tropic effector $\mathrm{CD} 8^{+} \mathrm{T}$ cells in vivo, carboxyfluorescein-labeled OT-I cells were transferred into mice maintained on a VAD diet from embryonic day 11 (long-term VAD) or control mice. The vitamin A status of animals was tracked by assessing serum levels of RBP-4 by enzyme-linked immunosorbent assay (ELISA) (Supplementary Figure 1 online). Three days after oral administration of ovalbumin (OVA), OT-I cells in the MLN of long-term VAD and control mice had proliferated extensively; however, CCR9 and $\alpha 4 \beta 7$ were only induced on MLN-primed OT-I cells in control mice (Figure 1a and $\mathbf{b}$ ). As expected from these results, OT-I cells efficiently localized to the SI epithelium of control but not to that of long-term VAD a
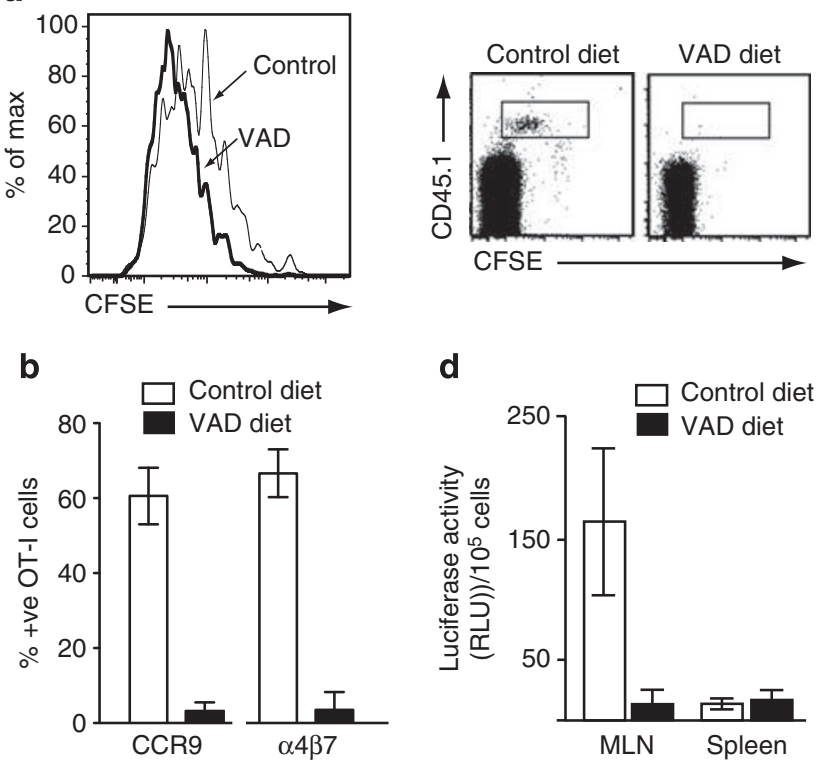

Figure 1 Vitamin A is essential for induction of $\mathrm{CC}$ chemokine receptor (CCR) 9 and $\alpha 4 \beta 7$ on activated T lymphocytes in vivo. (a-c) OT-I.CD45.1 cells were adoptively transferred into CD45.2 long-term vitamin Adeficient (VAD) mice or control mice. (a) CFSE dilution and (b) CCR9 and a4 37 expression on responding OT-I cells in the mesenteric lymph node (MLN) of mice kept on a conventional (white bar) or long-term VAD (black bar) diet 3 days after oral ovalbumin (OVA) administration. Results are (a) representative FACS plots from three experiments with two to three mice per group per experiment and (b) mean (s.d.) of four experiments with two to three mice per group per experiment. (c) FACS plots of CD8 ${ }^{+}$ intraepithelial lymphocytes in long-term VAD and control mice 3 days after OVA administration. Representative results from one experiment of three with two to three mice per group per experiment. (d) DR5.OT-I cells were adoptively transferred into CD45.1 long-term VAD or control mice. OVA and LPS were injected i.p., and 1 day later, mice received FTY720 to prevent OT-I.DR5 cell egress from secondary lymphoid organs. OT-I cells were sorted 3 days after immunization and assessed for luciferase activity. Results are the mean (s.d.) of three experiments, two to four mice per group per experiment. CFSE, carboxyfluorescein; FACS, fluorescence-activated cell sorting; i.p., intraperitoneal; LPS, lipopolysaccharide; RLU, relative luciferase units.

mice (Figure 1c). To determine whether $\mathrm{CD} 8^{+} \mathrm{T}$ cells receive RA signals when primed in the MLN, $\mathrm{CD}^{+}{ }^{+} \mathrm{T}$ cells from OT-I.DR5 mice, which express luciferase under control of three RA-responsive (DR5) elements, ${ }^{27}$ were adoptively transferred into control or long-term VAD mice. OVA and lipopolysaccharide were administered intraperitoneally to allow simultaneous T-cell priming in the spleen and MLN, and 1 day later, mice received FTY720 to prevent OT-I.DR5 cell egress from secondary lymphoid organs. Three days after immunization, OT-I.DR5 cells were sorted from the MLN and spleen and assayed for luciferase activity. OT-I.DR5 cells activated in the MLN of control mice displayed significantly enhanced luciferase activity compared with those activated in the spleen (Figure 1d). Importantly, luciferase activity was absent from OT-I.DR5 cells activated in the MLN of long-term VAD mice, confirming the RAR specificity of the transgene. Taken together, these results 
demonstrate a critical role for vitamin $\mathrm{A}$ in the generation of guttropic effector $\mathrm{CD}^{+} \mathrm{T}$ cells in vivo and also show that $\mathrm{CD} 8^{+}$ $\mathrm{T}$ cells receive enhanced RA signals when primed in the MLN compared with the spleen.

\section{Induction of gut-homing receptors by MLN DCs in vitro requires metabolism of serum-derived retinoids}

Although MLN DCs induce RA signals and RA-dependent CCR9 induction in responding $\mathrm{T}$ cells in vitro, ${ }^{26,27}$ it remains unclear whether the source of RA in these cultures derives from DC metabolism of retinoids present in fetal calf serum (FCS), or as recently suggested, ${ }^{28}$ whether MLN DCs pick up RA in the intestinal mucosa and this carriage of RA is sufficient to induce CCR9 on T cells. To assess these possibilities, MLN DCs were pulsed with OVA peptide and cultured together with carboxyfluorescein-labeled OT-I cells in X-VIVO10 medium, which supports lymphocyte culture in the absence of FCS (Figure 2a). Although OT-I cells primed in the R10 medium or in the $\mathrm{X}$-VIVO10 medium with 10\% FCS were induced to express gut-homing receptors, OT-I cells primed in the X-VIVO10 medium without FCS failed to express CCR9 and $\alpha 4 \beta 7$ (Figure 2a, data not shown). Thus, factor(s) in FCS are required for MLN DCs to induce gut-homing receptors on responding T cells in vitro. To determine whether the lack of CCR9 induction in the X-VIVO10 medium was due to a lack of retinol/retinal in these cultures, two different culture assays were set up. In the first assay, retinoids were destroyed in FCS by ultraviolet irradiation ${ }^{29}$ before addition to the medium, and under these conditions, CCR9 induction by MLN DCs was abrogated (Figure 2b). In the second assay, retinal alone was added to the X-VIVO10 cultures, and this resulted in the induction of CCR9 on responding OT-I cells (Figure 2b). Taken together, these results demonstrate that DC metabolism of retinal to RA, and not carriage of RA from the mucosa, underlies the ability of MLN DCs to generate gut-homing T cells in vitro.

\section{Imprinting of SI-LP CD103+ DCs is dependent on vitamin A}

A recent study by Yokota et al. ${ }^{14}$ demonstrated that total CD11c ${ }^{+}$ MLN cells from long-term VAD mice display reduced aldh1a2 expression and aldehyde dehydrogenase activity, indicating that vitamin A may have a potential role in regulating the ability of MLN DCs to metabolize retinol. To assess whether these findings extend to $\mathrm{CD}_{103}{ }^{+}$MLN DCs, we examined aldh1a2 expression and aldehyde dehydrogenase activity in $\mathrm{CD} 103^{+}$and $\mathrm{CD} 103^{-}$ MLN DCs of long-term VAD and control mice. Although the total number and percentage of $\mathrm{CD}_{103^{+}}$DCs in the MLN of long-term VAD mice remained unchanged compared with control mice (Supplementary Figure 2 online), $\mathrm{CD}_{103}{ }^{+} \mathrm{MLN}$ DCs from long-term VAD mice displayed a striking reduction in aldh1a2 expression and aldehyde dehydrogenase activity compared with $\mathrm{CD}_{103}{ }^{+}$MLN DCs from control mice (Figure 3a and $\mathbf{b}$ ). Aldehyde dehydrogenase activity and aldh1a2 expression was also dramatically reduced in SI-LP CD $103^{+}$DCs of long-term VAD mice along the entire length of the SI (Figure 3c, data not shown). Although colonic $\mathrm{CD} 103^{+} \mathrm{LP}$ DCs also displayed vitamin A-dependent retinol-metabolizing
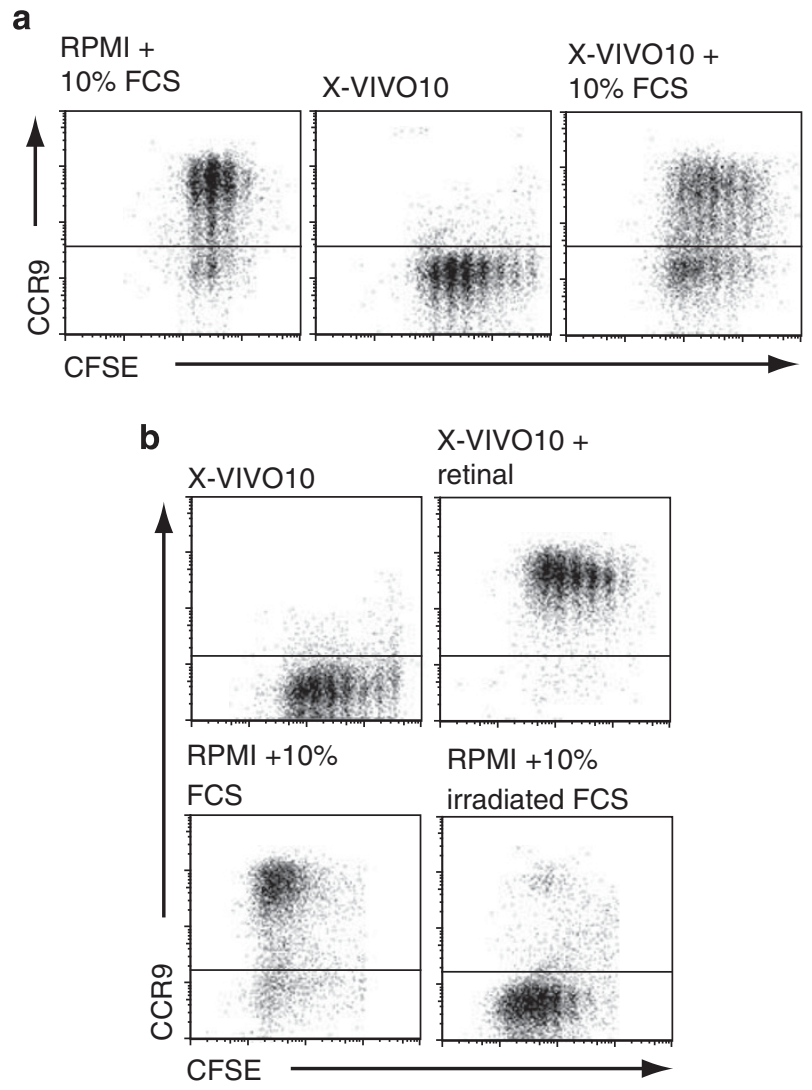

Figure 2 Induction of chemokine receptor (CCR)9 by mesenteric lymph node (MLN) dendritic cells (DCs) in vitro requires metabolism of exogenously derived retinol. (a, b) DCs were isolated from the MLN, pulsed with the ovalbumin (OVA) peptide ( $40 \mathrm{~nm}$ ), and cultured together with CFSE-labeled OT-I cells in RPMI or X-VIVO10 media with or without addition of fetal calf serum (FCS), UV-irradiated FCS, or retinal as indicated. CC chemokine receptor (CCR) 9 expression on responding OT-I cells was assessed 3 days later. (a) Results are representative FACS plots from one experiment of four performed. (b) Results are representative FACS plots from one experiment of four (UV irradiated) and three (retinal) performed. CFSE, carboxyfluorescein; UV, ultraviolet.

activity, aldh1a2 expression and aldehyde dehydrogenase activity was far lower in these cells compared with SI-LP CD $103^{+}$ DCs (Figure 3c, data not shown), consistent with their inability to induce CCR9 on effector T cells in vitro. ${ }^{5}$

Importantly, pre-DCs, that are the major precursors of SI-LP $\mathrm{CD}_{103}{ }^{+} \mathrm{DCs}^{24}$ as well as all major LN-resident DCs, ${ }^{30,31} \mathrm{did}$ not display aldehyde dehydrogenase activity (Supplementary Figure 3 online, data not shown). Collectively, these results demonstrate a critical and local role for vitamin A in imprinting $\mathrm{CD}_{103}{ }^{+}$DCs with retinol-metabolizing activity in the SI.

\section{RA signaling is observed in SI-LP CD103+ DCs in the steady state and induces retinol-metabolizing activity in DCs in vitro and in vivo}

As RA represents the major active metabolite of vitamin A, we next assessed whether RA may directly induce retinol-metabolizing activity in DCs. RA induced a significant increase in aldh1a2 expression and aldehyde dehydrogenase activity 

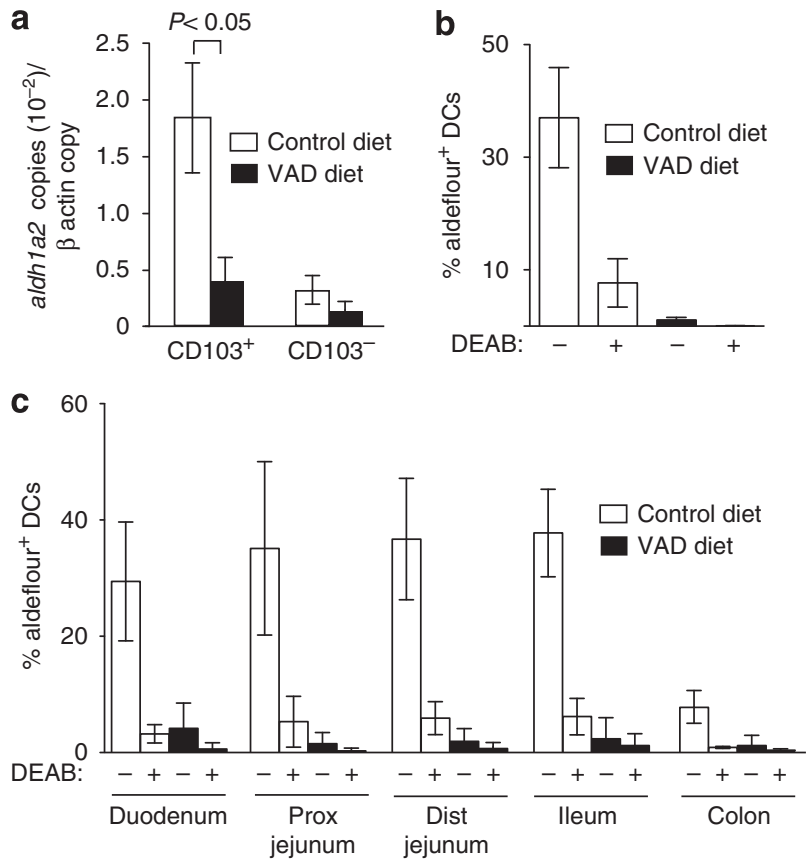

Figure 3 Vitamin $A$ is required for the selective imprinting of $C D 103^{+}$ dendritic cells (DCs) along the length of the small intestine (SI).

(a) $\mathrm{CD}_{103}{ }^{+}$and $\mathrm{CD} 103^{-} \mathrm{DC}$ s were sorted from the mesenteric lymph nodes (MLNs) of long-term vitamin A-deficient (VAD) mice and control mice and assessed for expression of aldh1a2 by real-time PCR. Mean (s.d.) from five to seven experiments with one to two mice per group per experiment. (b, c) Aldehyde dehydrogenase (ALDH) activity in (b) MLN and (c) SI and colonic lamina propria (LP) $\mathrm{CD}_{103}{ }^{+} \mathrm{DC}$ from control and long-term VAD mice was assessed by flow cytometry in the presence/ absence of the ALDH inhibitor diethylaminobenzaldehyde (DEAB). Mean (s.d.) of three to five experiments with one to three mice per group per experiment. Dist, distal; Prox, proximal.

in bone marrow-derived (BM)-DCs and imprinted these cells with an ability to generate CCR9 ${ }^{+}$effector T cells (Figure 4a-c and Supplementary Figure 4 online). To determine whether intestinal CD103 ${ }^{+}$DCs receive RA signals in vivo, SI-LP and colonic $\mathrm{CD}_{103}{ }^{+}$DCs were isolated from DR5 reporter mice and luciferase activity assessed by real-time PCR. SI-LP CD103 ${ }^{+}$ DCs constitutively expressed luciferase at significantly higher levels than did colonic CD103 ${ }^{+}$DCs (Figure 4d). Taken together, these results demonstrate that RA can have a direct role in imprinting DCs with the ability to metabolize retinol and that SI-LP CD $103^{+}$DCs receive enhanced RA signaling compared with colonic $\mathrm{CD} 103^{+}$DCs in the steady state.

To assess whether the reduced imprinting of colonic compared with SI-LP CD103 ${ }^{+}$DCs might be due to a reduced ability of colonic $\mathrm{CD} 103^{+} \mathrm{DCs}$ to respond to RA, or to reduced retinoid levels in the colon vs. the SI, we first assessed RAR expression in intestinal $\mathrm{CD}_{103}{ }^{+} \mathrm{DCs}$. RAR $\alpha$ and RAR $\gamma$ expression was comparable in SI-LP and colonic CD103 ${ }^{+}$DCs (Figure 5a), whereas RAR $\beta$ was barely detectable. In contrast, analysis of retinol/retinal levels in a range of intestinal and extra-intestinal tissues demonstrated significantly higher concentrations of retinol and retinal in the SI and draining MLN than in the
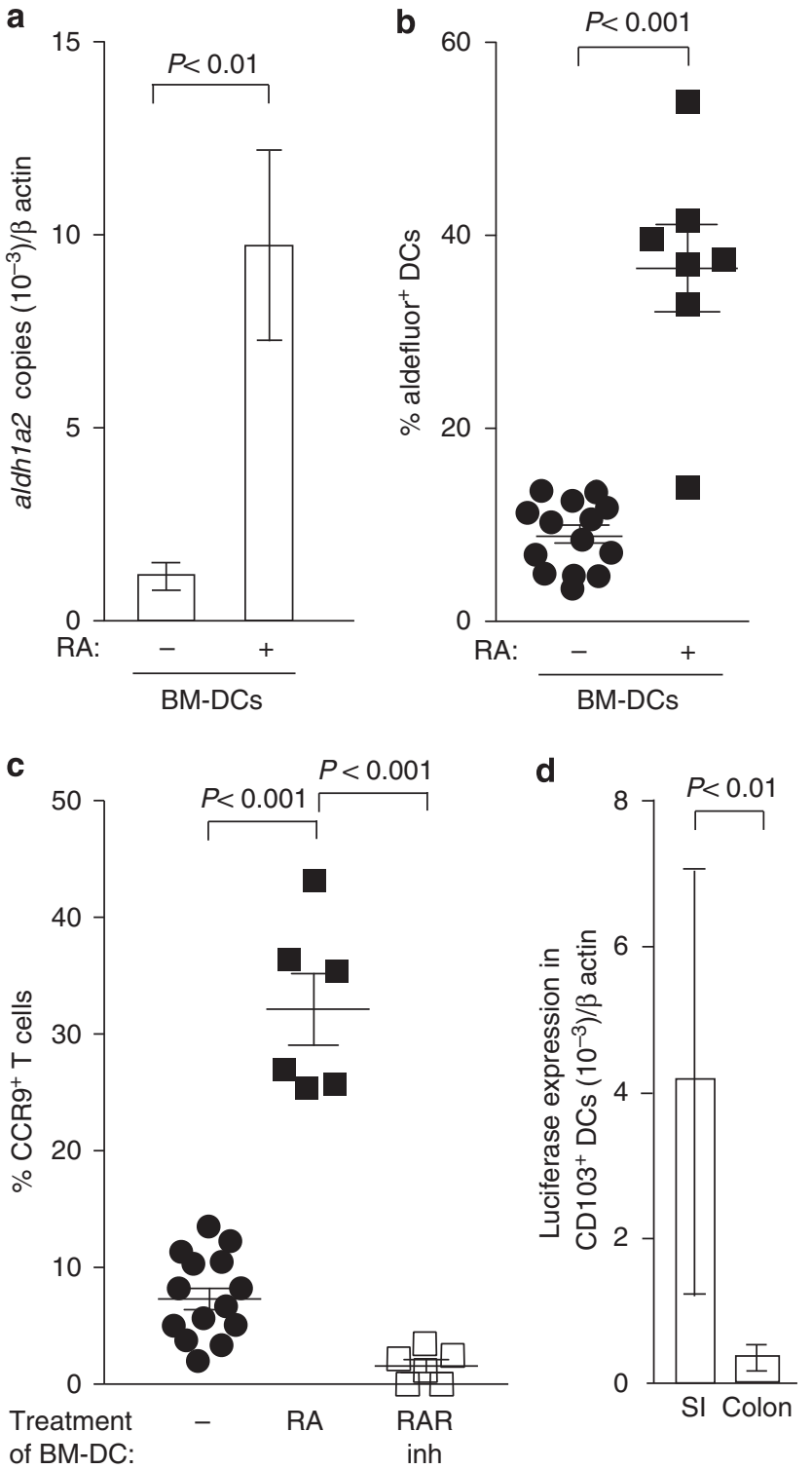

Figure 4 Retinoic acid (RA) induces retinol-metabolizing activity in dendritic cells (DCs). (a) Bone marrow-derived (BM)-DCs were treated with RA (20 nM) for 2 days, and aldh1a2 expression on sorted CD11 $\mathrm{C}^{+}$ MHCII int cells was assessed by real-time PCR. Mean (s.d.) of four experiments. (b) BM-DCs were incubated with RA as described above, and aldehyde dehydrogenase activity was determined by flow cytometry. Each symbol represents cells from a single well. Data obtained from six separate experiments are shown. (c) BM-DCs were incubated with RA (20 nM) with/without the RA receptor inhibitor (inh) LE540 (2 $\mu \mathrm{m})$ for 2 days, pulsed with ovalbumin (OVA), washed and incubated together with OT-I cells. CC chemokine receptor (CCR) 9 expression on OT-I cells was assessed 3.5 days later. Symbols are results obtained from independent wells from three different experiments in which each well contained BM-DCs treated separately with RA. (d) CD103+ DCs were sorted from the lamina propria (LP) of the small intestine (SI) and colon of DR5 mice and assessed for luciferase activity by real-time PCR. Mean (s.d.) of three experiments with sorted cells from two mice per organ per experiment. RAR, RA receptor.

colon (Figure 5b, data not shown). Collectively, these results suggested that enhanced levels of the RA precursors, and not selective responsiveness of SI-LP CD $103^{+}$DCs to RA, underlie 

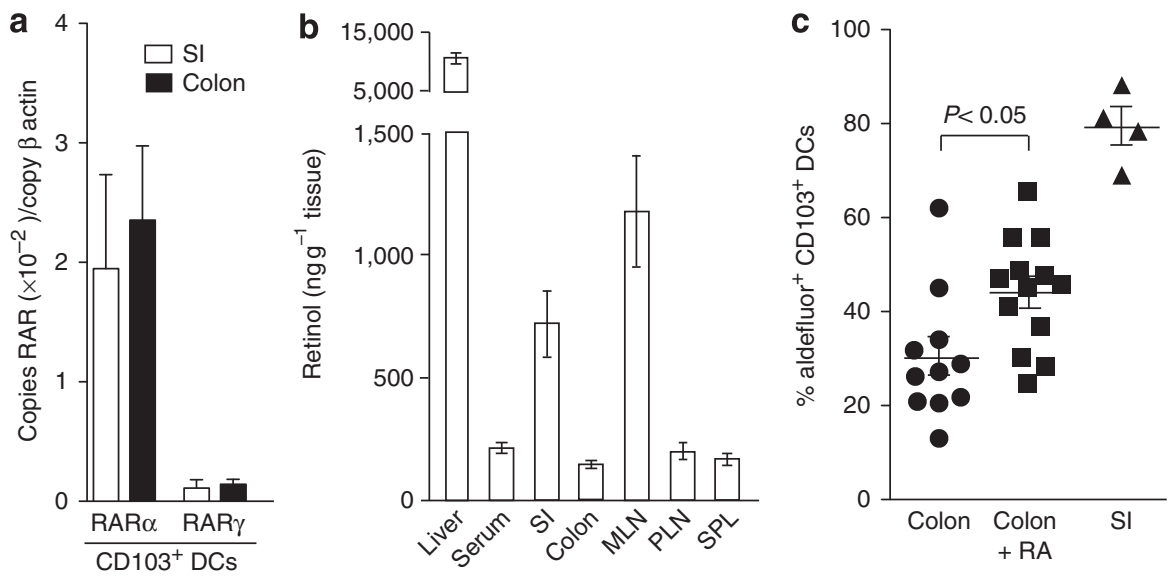

Figure 5 Retinol levels are higher in the small intestine (SI) than in the colon and correlate with enhanced RA signaling in the small intestinal lamina propria (SI-LP) CD103+ DCs. (a) RA receptor (RAR) $\alpha$ and RAR $\gamma$ mRNA levels in sorted SI and colonic CD103+ DCs. Mean (s.d.) of three to five experiments with sorted cells from one to two pooled mice per experiment. (b) Retinol levels in tissues. Mean (s.d.) of retinol determinations from tissues of eight individual mice. (c) RA (filled boxes) or carrier (filled circles, filled triangles) was administered intra-rectally into mice as described in the "Methods" section, and aldehyde dehydrogenase activity in SI and colonic LP CD11b+ CD103+ DCs was assessed by flow cytometry. Each symbol represents an individual mouse. DC, dendritic cell; MLN, mesenteric lymph node; PLN, peripheral lymph node; RA, retinoic acid; SPL, spleen.

the selective imprinting of SI-LP CD103 ${ }^{+}$DCs. To determine whether the availability of RA is a potential rate-limiting factor in imprinting colonic $\mathrm{CD} 103^{+}$DCs, RA was administered intrarectally into C57BL/6 mice. Administration of RA induced a significant increase in aldehyde dehydrogenase activity in colonic $\mathrm{CD} 103^{+} \mathrm{DCs}$ (Figure 5c), indicating that local RA levels are one factor regulating the imprinting of DCs in vivo. Notably, the percentage of colonic $\mathrm{CD} 103^{+}$DCs expressing aldehyde dehydrogenase activity after RA treatment failed to reach levels found in SI CD $103^{+}$DCs of control mice, suggesting that other factors in the SI may synergize with RA to enhance the activity of SI $\mathrm{CD}_{103}{ }^{+} \mathrm{DCs}$. However, we cannot rule out the possibility that the concentrations of RA used and administration regime for intra-rectal delivery were suboptimal for colonic CD103 ${ }^{+}$DC imprinting. Taken together, these data demonstrate that RA can directly induce retinol-metabolizing activity in DCs and suggest that the high retinol/retinal levels in the SI at least partially underlie selective imprinting of $\mathrm{CD} 103^{+} \mathrm{DCs}$ at this site.

\section{Dietary vitamin A is not required for imprinting SI-LP CD103+ DCs with retinol-metabolizing activity}

As vitamin A is obtained through diet and the SI and MLN contained significantly higher retinol and retinal levels than did other tissues outside the liver (Figure $\mathbf{5 b}$ and data not shown), we hypothesized that dietary vitamin A was critical for the selective imprinting of SI-LP CD $103^{+}$DCs. To address this possibility, 5-7-week-old mice were transferred to a VAD or maintained on a conventional diet for 4 weeks (4-week VAD). This time period is too short to effect liver stores or systemic levels of retinol; however, it is sufficiently long enough to allow a complete turnover of the epithelial and SI-LP CD $103^{+}$DC compartment in the absence of dietary retinol. ${ }^{5,32}$ Mice kept on a 4-week VAD diet showed a marked reduction in retinol levels in the MLN, whereas retinol levels in the liver, spleen, peripheral lymph nodes (LNs), and serum remained unchanged (Figures 6a and $7 \mathbf{b}$ ), thus demonstrating that dietary retinol accounts for much of the enhanced retinol levels observed in the MLN. Strikingly, under these conditions, MLN CD $103^{+}$DCs still expressed high levels of aldh $1 a 2$ and aldehyde dehydrogenase activity, whereas aldehyde dehydrogenase activity in SI-LP CD $103^{+}$DCs was only partially reduced (Figure $\mathbf{6 b}-\mathbf{d}$ ). Taken together, these results demonstrate that dietary retinol intake, although likely contributing to DC imprinting in the steady state, is not essential to maintain selective imprinting of SI-LP CD $103^{+}$DCs.

\section{Bile contains high levels of retinol and imprints DCs with the ability to generate gut-tropic effector $T$ cells}

Despite the reduction of retinol in the MLN of 4-week VAD mice, retinol levels remained significantly higher than in other tissues, indicating that even in the absence of dietary vitamin A, the SI mucosa may receive additional sources of retinol (Figure 6a). Consistent with this possibility, SI-LP CD103 ${ }^{+}$DCs from DR5 mice maintained on a 4-week VAD diet continued to receive enhanced RA signals compared with colonic $\mathrm{CD} 103^{+}$ DCs (Figure 7a). Notably, the liver, the retinol levels of which remained unchanged in 4-week VAD mice (Figure 6a), is also the source of bile acids that have an essential role in emulsifying dietary fats and fat-soluble vitamins in the SI. Bile drains from the liver through the gall bladder and the common bile duct into the duodenum, with the majority of bile salts being recycled after re-absorption in the ileum through the entero-hepatic circulation. As retinoids have been reported to be present in the bile of guinea pigs, rats, and chicks, ${ }^{33-36}$ we hypothesized that the selective imprinting of CD103 ${ }^{+}$DCs in the SI may result from the continual supply of retinol and its derivatives from the liver through the bile. Consistent with this hypothesis, retinol levels in the bile of mice kept on a conventional or a 4-week VAD diet were significantly higher than in the serum (Figure 7b). 

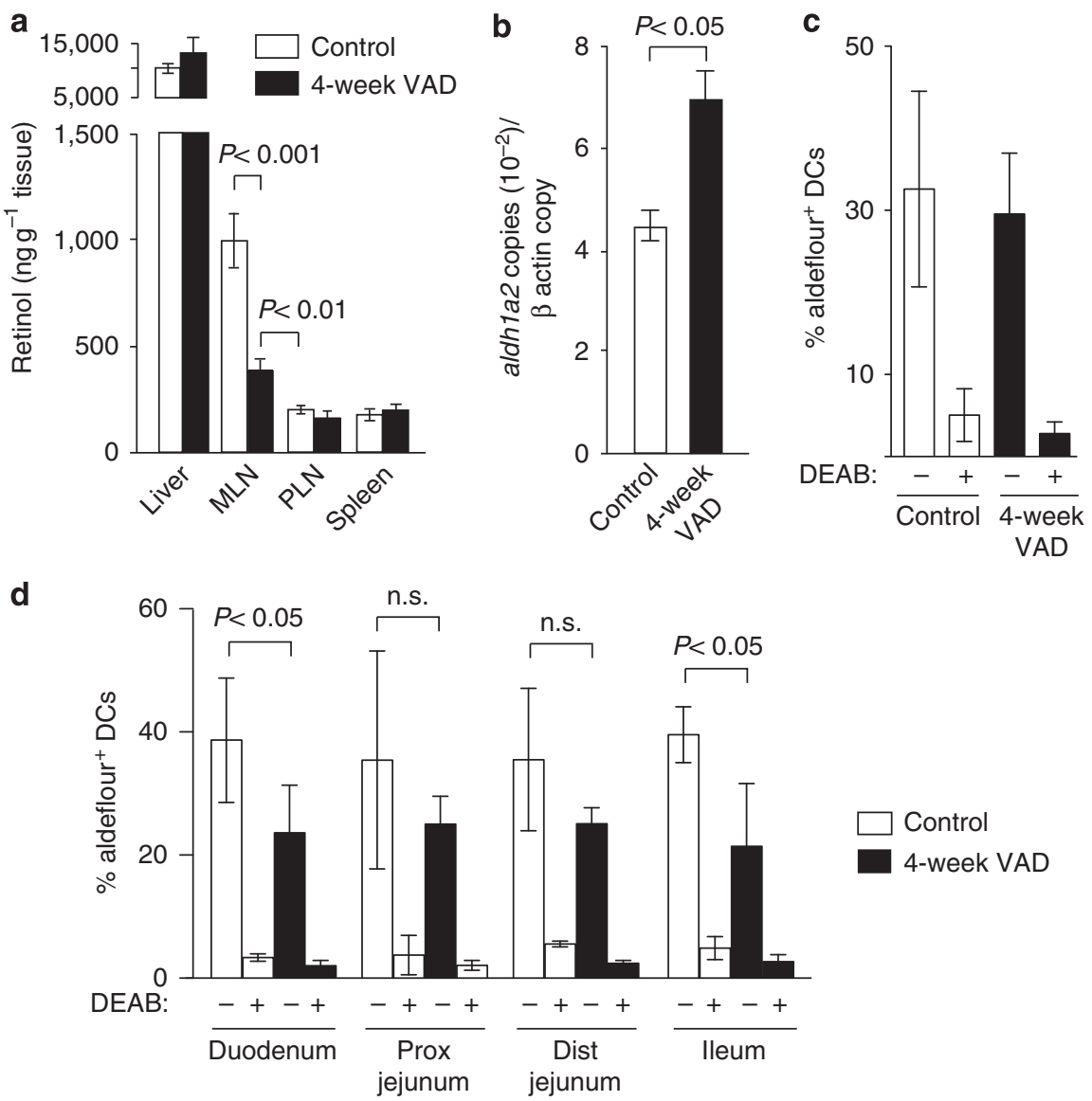

Figure 6 Dietary vitamin A is not required for imprinting of small intestinal CD103+ dendritic cells (DCs). (a) Retinol levels are higher in the mesenteric lymph nodes (MLNs) than in the peripheral lymph nodes (PLN) and spleen of 4-week vitamin A-deficient (VAD) mice. Mean (s.d.) of 4-15 individual mice. (b) Aldh1a2 expression in sorted CD103+ MLN DCs from 4-week VAD and control mice was assessed by real-time PCR. Data are obtained from one representative experiment of two performed using two to three pooled mice per group per experiment. (c, d) Aldehyde dehydrogenase (ALDH) activity in (c) MLN and (d) small intestinal lamina propria (SI-LP) CD103+ DCs from 4-week VAD and control mice was assessed by flow cytometry in the presence/absence of the ALDH inhibitor diethylaminobenzaldehyde (DEAB). Mean (s.d.) of three to five experiments (two experiments for MLN control DEAB) using one to two pooled mice per group per experiment. Dist, distal; n.s., not significant; Prox, proximal.

To assess whether bile induces RA signaling, BM-DCs from DR5 mice were incubated with $0.5 \%$ bile, and luciferase activity was assessed 2 days later (Figure 7c). This concentration of bile did not affect the total number or viability of BM-DCs in the cultures as assessed by flow cytometry (data not shown). Bile from mice maintained on a conventional but not long-term VAD diet induced luciferase expression in BM-DCs (Figure 7c, data not shown). Importantly, bile from mice maintained on a conventional or 4-week VAD diet induced aldehyde dehydrogenase activity and aldh1a2 expression in BM-DCs (Figure 7d-e and Supplementary Figure 4a online), which was dependent on RA signaling as it was blocked by the pan-RAR inhibitor, LE540. Finally to determine whether bile-conditioned DCs could generate CCR9 ${ }^{+}$effector T cells, DCs were incubated with bile in the presence/absence of LE540, washed, pulsed with OVA, and incubated with OT-I cells. BM-DCs conditioned with bile or 4-week VAD bile induced CCR9 on responding OT-I cells, whereas OT-I cells primed by BM-DCs that had been conditioned with bile in the presence of LE540 failed to express CCR9 (Figure 7 f and Supplementary Figure $4 \mathbf{b}$ online). Collectively, these results indicate that in the absence of dietary vitamin $\mathrm{A}$, bile-derived retinoids contribute to the selective imprinting of SI-LP CD $103^{+}$DCs.

\section{DISCUSSION}

$\mathrm{CD}_{103}{ }^{+} \mathrm{DCs}$ are the major migratory DC population in the SI and have a central role in initiating immune responses to luminal antigen in draining MLN. Both SI-LP and MLN CD $103^{+}$DCs are imprinted with the ability to metabolize retinol, a property that underlies their enhanced capacity to generate gut-homing effector T cells and induce FoxP3 ${ }^{+}$T-cell differentiation. ${ }^{3,6,8,10-12}$ In this study, we demonstrate that imprinting of SI-LP CD103 ${ }^{+}$ DCs occurs locally in the SI mucosa and is critically dependent on vitamin A. Our results further show that dietary vitamin A is not essential for imprinting and suggest a role for liver-derived bile retinoids in the selective imprinting of $\mathrm{CD}_{103}{ }^{+}$DCs.

A recent study by Feng et al., ${ }^{37}$ based primarily on in vitro work, suggested that RA induces CCR9 expression and 

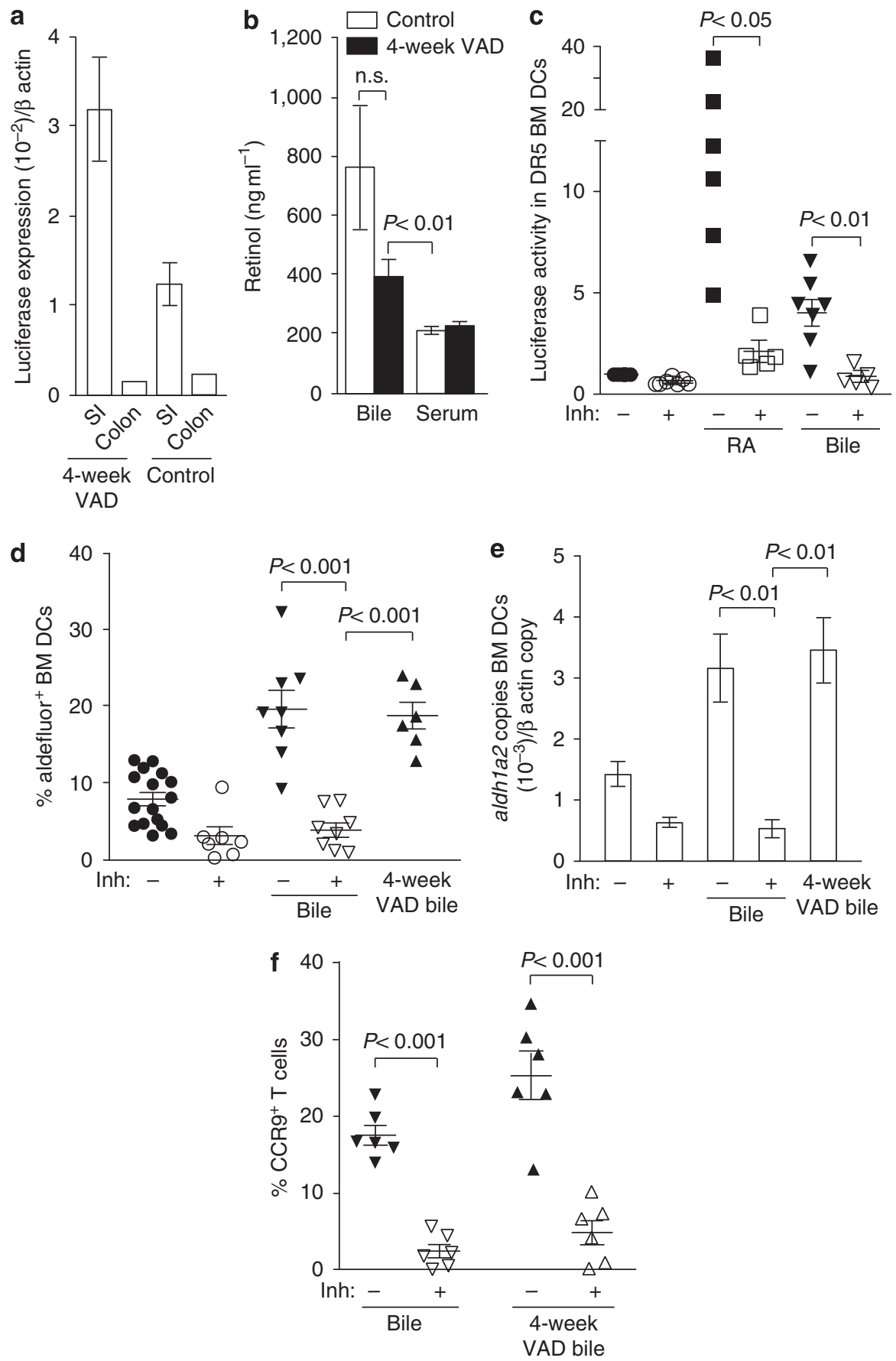

Figure 7 Bile contains high levels of retinol and imprints bone marrow-derived dendritic cells (BM-DCs) with the ability to generate CC chemokine (CCR) $9^{+}$T cells. (a) Luciferase expression in small intestinal lamina propria (SI-LP) and colonic CD103+ DCs from DR5 mice kept on a 4-week vitamin A-deficient (VAD) or control diet. Results are the mean (s.d.) of 3 (4-week VAD) or 2 (control diet) independent experiments using pooled SI-LP $\mathrm{CD}_{103}{ }^{+} \mathrm{DCs}$ from 2 mice per experiment, or from 1 experiment using pooled colonic $\mathrm{CD} 103^{+} \mathrm{DCs}$ from 3 mice. (b) Bile and serum retinol levels in control and 4-week VAD mice. Mean (s.d.) of 7-12 individual mice (serum) or from 7-12 samples from 1-3 pooled mice per sample (bile). (c) BM-DCs from DR5 mice were incubated for 2 days with retinoic acid (RA) (20 nм) or bile ( $0.5 \%)$ with/without the pan RA receptor inhibitor (inh) LE540 ( $2 \mu \mathrm{M})$. Luciferase activity $/ 10^{5} \mathrm{MHCII}{ }^{+} \mathrm{CD} 11 \mathrm{c}^{+}$cells was determined and normalized in each experiment to the control. Each symbol represents a single experiment with separate bile samples per experiment. (d) Aldehyde dehydrogenase activity and (e) aldh1a2 expression in $\mathrm{CD} 11 \mathrm{C}^{+} \mathrm{MHCl} \mathrm{I}^{\text {int }} \mathrm{BM}-$ DCs after treatment with the indicated compounds. (d) Each symbol represents data obtained from individual wells in which BM-DCs were incubated with bile samples from different mice. Data are mean (s.d.) of four to six independent experiments. (f) BM-DCs were incubated with bile (0.5\%) in the presence or absence of RAR inhibitor (inh) for 2 days, washed, pulsed with ovalbumin (OVA), and incubated together with OT-I cells. CCR9 expression on responding cells was assessed 3.5 days later by flow cytometry. Each symbol represents data from individual wells in which BM-DCs were preincubated with bile samples obtained from different mice. Mean (s.d.) of two to three experiments. n.s., not significant. 
retinol-metabolizing activity on DCs in the bone marrow thus generating a specific intestinal homing DC subset. Although our results also demonstrate a critical role for vitamin A in the generation of retinol-metabolizing SI-LP CD $103^{+}$DCs, they strongly argue against this model. Thus, we found that the number of CD103+ MLN DCs was not reduced in longterm VAD mice, as might be expected if RA was required for CCR9-dependent recruitment of DCs into the SI. Indeed, in CCR9 KO mice, SI-LP and MLN CD103 ${ }^{+}$DCs are found in normal numbers and display comparable aldehyde dehydrogenase activity to that observed in wild-type mice (K.K., unpublished observation). Second, using RA-responsive element (DR5) reporter mice, we show that $\mathrm{CD} 103^{+} \mathrm{DCs}$ constitutively receive RA signals in the SI and at significantly higher levels than in the colon. Most importantly, we demonstrate that pre-DCs in the SI-LP, which are the major precursors of SI-LP CD $103^{+}$DCs, ${ }^{25}$ lack retinol-metabolizing activity. Collectively, these results strongly indicate that RA functions locally within the SI to imprint $\mathrm{CD}_{103}{ }^{+} \mathrm{DCs}$. Although we provide in vitro evidence that RA can directly imprint DCs with retinol-metabolizing activity, our results do not exclude that potential indirect effects of RA may contribute to SI-LP CD $103^{+}$DCs imprinting in vivo; nor can we rule out that indirect effects of VAD deficiency may contribute to the block in DC imprinting observed in these mice.

A striking observation made from the current study was that SI-LP CD $103^{+}$DCs isolated from mice deprived of dietary retinol, but with normal systemic retinol levels, still received high RA signals and remained imprinted with retinol-metabolizing activity. Bile drains from the liver through the gall bladder and common bile duct into the duodenum. In this study, we show that bile from conventionally fed and 4-week VAD mice also contains retinol and at significantly higher concentrations than found in serum and other tissues (outside the liver). Importantly, bile from these mice induced RA signals in DCs and imprinted them with retinol-metabolizing activity and the ability to generate gut-tropic effector $\mathrm{T}$ cells. These results strongly suggest that liver-derived retinol and its metabolites are transported through the bile, bathing the SI, where they contribute to SI-LP CD $103^{+}$ DC imprinting. Although the current study focused on the role of vitamin A in imprinting CD103 ${ }^{+}$DCs, two recent studies have demonstrated that MLN but not peripheral LN stromal cells also possess the enzymatic machinery to generate RA, and thus may contribute to gut-tropic T-cell generation in the MLN in vivo. ${ }^{38,39}$ Thus, it will be of interest to determine whether dietary and/or bile-derived retinoids are also involved in the selective imprinting of MLN stromal cells.

Several previous studies have suggested a central role for bile constituents in maintaining intestinal immune homeostasis. For example, mice deficient in the major bile acid farnesoid $\mathrm{X}$ receptor show increased bacterial counts and disturbed epithelial barrier function in the ileum, ${ }^{40}$ increased proinflammatory cytokines in the colon, and exacerbated inflammation in 2,4,6 trinitrobenzene sulfonic acid (TNBS) colitis. ${ }^{41}$ Furthermore, farnesoid $\mathrm{X}$ receptor ligands repress lipopolysaccharide-induced inflammatory cytokine expression in intestinal LP macrophages. ${ }^{41}$ Both farnesoid
$\mathrm{X}$ receptor and RAR form a heterodimer with RXR to function as ligand-induced transcription factors bound to DNA target genes. In this regard, it will be of considerable interest to determine the functional interplay between bile-induced farnesoid X receptor-/RXR and RAR/RXR signaling in the modulation of SI-LP CD $103^{+}$DC function.

In summary, our results describe a novel role for vitamin A and bile in local imprinting of SI-LP CD103 ${ }^{+}$DCs, and thus in shaping the unique characteristic of SI immune responses.

\section{METHODS \\ Mice}

C57BL/6, C57BL/6.CD45.1, OT-1, OT-I.CD45.1, DR5, and DR5.OT-I mice ${ }^{27}$ were bred and maintained at the Biomedical Center Animal Facility (Lund University, Sweden). Mice were used between 6 and 14 weeks of age. Long-term VAD mice were generated by placing pregnant mice on a VAD diet (TD88407, Harlan Laboratories, Madison, WI) starting at day 11 of gestation, and pups were maintained on this diet until at least 8 weeks of age. Vitamin A deficiency was confirmed by analyzing serum levels of RBP-4 by ELISA or liver retinol levels upon killing. Protocols for the ELISA and quantitation of retinol are described below. Control mice were maintained on a vitamin A-sufficient diet (TD08536, Harlan Laboratories). For generating 4-week-VAD mice, 5-7-week-old littermates were placed on a VAD (TD88407) or a vitamin A-sufficient diet (TD08536) for 4 weeks. For in vivo RA administration, anesthetized animals were injected intrarectally with RA $(10 \mu \mathrm{M})$ or vehicle (dimethyl sulfoxide) in phosphatebuffered saline (PBS) 64, 40, 26, and $16 \mathrm{~h}$ before the killing. All animal experiments were performed after obtaining approval from the Lund/ Malmö Animal Ethics Committee.

\section{Antibodies and reagents}

The following antibodies were used for flow cytometry: PE or bio-CD103 (M290), PE- $\alpha 4 \beta 7$ (DATK32), APC-CD11c (N418), PE or bio-rat IgG2a (RG7/1.30), bio-IgG2b isotype, bio-rat IgG2b (G15-337), streptavidin-PE, streptavidin-PECy7, PE-CD45.1 (A20), PE-Cy7-CD45 (Ly-5), streptavidin-APC, bio-CD8 $\beta$ (53-5.8), PE-V $\beta$ 5.1.5.2 (MR9-4) (all obtained from BD Biosciences, Franklin Lakes, NJ); Alexa700-CD11c (N418), Pacific Blue-MHC Class II (M5/114.15.2), Alexa700-CD45.1 (A20) (all obtained from BioLegend, San Diego, CA); PE and APC-A750-CD45.2 (104), APC-V 22 (B20.1), and Pacific Blue-CD8 $\alpha$ (53-6.7) (all purchased from eBioscience, San Diego, CA); streptavidin-QD605 (Invitrogen, Carlsbad, CA); anti-FcRII/III (2.4G2) and anti-CCR9 antibody (7E7, kind gift from O. Pabst ${ }^{42}$ ) were purified from hybridomas. The OT-I peptide (SIINFEKL) was obtained from Innovagen, Lund, Sweden. OVA grade VII, DNase I, collagenase type IV and VIII, and lipopolysaccharide (Escherichia coli, serotype 055:B5) were from Sigma-Aldrich, St Louis, MO. FTY720 was obtained from Cayman Chemical, Ann Arbor, MI. The $\mathrm{X}$-VIVO10 medium was from Cambrex (Walkersville, MD).

\section{Cell isolation}

Cells were prepared from the SI and colon as described previously. ${ }^{5}$ In brief, the intestinal tissue was rinsed, Peyer's patches and fat were excised, and the tissue was cut open longitudinally and into $\sim 0.5 \mathrm{~cm}$ pieces. Tissue pieces were incubated with $2 \mathrm{~mm}$ EDTA to remove the epithelium and intraepithelial lymphocytes. Intraepithelial lymphocytes were enriched from this fraction on a 40:70\% percoll gradient. SI-LP DCs were isolated from remaining tissue pieces by collagenase VIII $(250 \mu \mathrm{g} / \mathrm{ml})$ digestion in the presence of $\mathrm{CaCl}_{2}(50 \mathrm{~mm})$. Where indicated, SI was divided into the duodenum (first $2.5 \mathrm{~cm}$ after the pylorus), the ileum (distal $4 \mathrm{~cm}$ of the SI), and the remaining jejunum was divided equally into two portions, the proximal and the distal jejunum. For isolation of LNT cells and DCs, LNs were cut and filtered directly through a 70-mm cell strainer or incubated with collagenase IV $(500 \mu \mathrm{g} / \mathrm{ml})$ and DNAse I 
(50 Units $/ \mathrm{ml}$ ) for $30 \mathrm{~min}$ at $37^{\circ} \mathrm{C}$ before filtering, respectively. CD8 $\beta^{+}$ cells were enriched to $>85 \%$ purity using bio-anti-CD8 $\beta$ (53-5.8), followed by streptavidin-conjugated MACS beads (Miltenyi Biotech, Bergisch Gladbach, Germany).

\section{Cell purification and flow cytometry}

DCs were sorted directly (LP preparations) or enriched using antiCD11c-conjugated MACS beads (Miltenyi Biotech) (LN preparations) before sorting. Dead cells were excluded using 7-amino-actinomycin D (Sigma-Aldrich) or propidium iodide (Invitrogen). Sorted DC fractions were routinely $95-99 \%$ pure. Flow cytometry analysis was performed using FACSAria, FACSCalibur, or FACS LSRII (BD Biosciences) and analyzed using FlowJo software (Tree Star, Ashland, OR). Reagents used for flow cytometry are described in the online Supplementary Materials online.

\section{Adoptive transfers}

Carboxyfluorescein-labeled OT-I or OT-I.DR5 CD8 $\beta^{+}$T cells were injected intravenously $\left(3-5 \times 10^{6}\right.$ per mouse) into C57BL/6 mice. One day later, mice received OVA by gavage $(50 \mathrm{mg})$ or by intraperitoneal injection $(5 \mathrm{mg})$. Where indicated, mice received lipopolysaccharide intraperitoneally $(25 \mu \mathrm{g})$ together with OVA and/or FTY720 $(20 \mu \mathrm{g}$, intraperitoneally) $24 \mathrm{~h}$ after OVA administration.

\section{BM-DCs}

BM-DCs were generated as described previously. ${ }^{43}$ For determinations of RA signaling, day 9 BM-DCs from DR5 mice $\left(2.5 \times 10^{5}\right)$ were seeded into 24-well ultra-low attachment plates (Costar-Corning, Amsterdam, Netherlands) and incubated as indicated for 2 days. Luciferase activity was determined using the luciferase assay system kit (Promega, Madison, WI) and a Glomax luminometer (Promega) according to the manufacturer's instructions. For ALDEFLUOR measurements, day 6 BM-DC cultures were incubated with RA or bile as indicated for 2 days and then analyzed by flow cytometry as described below. Live $\mathrm{MHCII}^{+} \mathrm{CD} 11 \mathrm{c}^{+}$ cells were sorted from the same cultures for PCR analysis. RA or bile at the concentrations used had no significant effect on the total number or proportion of $\mathrm{MHCII}^{+} \mathrm{CD} 11 \mathrm{c}^{+}$cells in these cultures, or on the expression levels of MHCII (data not shown).

\section{In vitro DC-T-cell cultures}

MLN DCs or day 8 BM-DCs were pulsed with OVA for $2 \mathrm{~h}$ at $37^{\circ} \mathrm{C}$, washed extensively, and incubated with OT-I cells in flat bottom 96-well plates (Thermo Fisher Scientific, Waltham, MA) in $250 \mu \mathrm{l} \mathrm{R} 10$ media at a 1:2 ratio $\left(2.5 \times 10^{4} \mathrm{DCs}\right.$ with $5 \times 10^{4} \mathrm{~T}$ cells). Responding OT-I cells were analyzed after 3.5 day by flow cytometry.

\section{Retinol measurements}

Tissues were weighed and stored at $-80^{\circ} \mathrm{C}$ before analysis. For bile collection, the gallbladder was removed, placed in an Eppendorf tube, and punctured with a 30-G needle. The gallbladder was fixed with one edge between the tube and the lid, and centrifuged for $1 \mathrm{~min}$ at $20,000 \mathrm{~g}$. Bile was stored at $-20^{\circ} \mathrm{C}$ before analysis. Tissues were homogenized in ice-cold PBS using a motorized homogenizer (Pellet grinder, Kontes, Vineland, NJ). Retinoids were extracted from the tissue homogenate by adding 2 volumes of ice-cold acetonitrile (containing a ${ }^{13} \mathrm{C}$-labeled stable isotope of atRA as the internal standard) per volume of tissue homogenate (e.g., $200 \mathrm{mg}$ tissue homogenate; $400 \mu \mathrm{l}$ acetonitrile) shaken for $15 \mathrm{~min}$ in an orbital shaker and centrifuged $\left(10 \mathrm{~min}, 10^{\circ} \mathrm{C}, 2,700 \mathrm{~g}\right)$. The entire procedure was performed under red light. An aliquot of $75 \mu \mathrm{l}$ was injected into a 4000 Q-TRAP LC-MS/MS, triple quadrupole mass spectrometer with APCI ionization (Applied Biosystems, Foster City, CA). Chromatographic analysis and mass spectrometric conditions were as described previously, ${ }^{44}$ except that the separating column was a Supelco ABZ Plus, $70 \times 3 \mathrm{~mm}^{2} \mathrm{ID}, 3 \mu \mathrm{m}$ particles (Supelco, Bellefonte, $\mathrm{PA}$ ).

\section{Mouse RBP-4 ELISA}

ELISA plates (Maxisorb, NUNC, Roskilde, Denmark) were coated overnight with serum (1:1,666 to 1:5,000) or recombinant mouse RBP-4
(1:500 to 1:1,66,000, R\&D Systems, Minneapolis, MN) diluted in PBS. Plates were blocked with blocking buffer (PBS and 1\% bovine serum albumin) for $1 \mathrm{~h}$ at room temperature, washed with PBS and $0.05 \%$ Tween 20, and incubated with biotinylated sheep anti-mouse RBP-4 $(0.5 \mathrm{mg} / \mathrm{ml}, \mathrm{R} \& \mathrm{D}$ Systems $)$ in blocking buffer for $1.5 \mathrm{~h}$ at room temperature. After washing, streptavidin-coupled peroxidase (1:4,000, BioLegend) was applied and plates incubated for $45 \mathrm{~min}$ at room temperature. BD OptEIA TMB Substrate Reagent Set (BD Bioscience) was used for color development, the reaction stopped with $2 \mathrm{~N}$ sulfuric acid and absorbance was measured at $450 \mathrm{~nm}$ using a Emax plate reader (Molecular Devices, Sunnyvale, CA).

\section{Real-time PCR}

Total RNA was isolated from sorted cells using the RNeasy Micro Kit (Stratagene, Santa Clara, CA). cDNA was generated using Superscript III First-Strand Kit (Invitrogen) with both random hexamers and pT-primer present and used as a template for quantitative real-time PCR. Quantitative PCR was performed on a MyiQ Single-Color Real-Time PCR Detection System (Bio-Rad, Hercules, CA), using the Power Sybr Green PCR Master Mix (Applied Biosystems, Warrington, UK) or SsoFAST EvaGreen SuperMix (Bio-Rad) according to the manufacturer's instructions. The following primers were used: aldh1a2 forward 5'CAGATGCTGACTTGGACTAC3'; aldh1a2 reverse 5'ATAAGCTCCAGGACTTTGTT3'; $\beta$-actin forward 5'CCGGG ACCTGACAGACTA3 ${ }^{\prime} ; \beta$-actin reverse $5^{\prime}$ GTTTCATGGATGCCACAGG $\mathrm{AT}^{\prime}$; RAR $\alpha$ forward 5'CGAATCTGCACGCGGTACACGCCT3'; RAR $\alpha$ reverse 5'GTGCTTCCAGCAGCGGCTCTTG3'; RAR $\beta$ forward 5'ACT GACCTTGTGTTCACCTTTGCCA3'; RAR $\beta$ reverse 5' GGTCGTCGT TTTCTAATGTAAATCT3'; $R A R \gamma$ forward 5'CAGTATGTAGGAGG GACTCTCTGCA3'; RAR $\gamma$ reverse 5'TCCTTGGGGAGGTCAGGCT GGCCC3'; firefly luciferase forward 5'CGTTCGTCACATCTCATCTCAT CTACCTC3'; firefly luciferase reverse 5'CCACATATCAAATATCCGAG TGT3'; and RDH10 forward 5'GTTTCTGGACATCACCTTCT3'; RDH10 reverse 5'GTAATCCTCAACTCCAGCA3'. For Figure 7a, samples were first amplified for 12 cycles by PCR using the following primers: $\beta$-actin forward 5'CCGGGACCTGACAGACTA3'; $\beta$-actin reverse 5'ACGGATGTCAACGTCACACTTC3'; and firefly luciferase forward 5'CAGTCGATGTACACGTTCGTCAC3'; firefly luciferase reverse 5'CCACATATCAAATATCCGAGTGT3'. For subsequent quantification by nested PCR, the following primers were used: $\beta$-actin forward 5'GAGAGGGAAATCGTGCGTGAC3'; $\beta$-actin reverse 5'GTTT CATGGATGCCACAGGAT3'; and firefly luciferase forward 5'CGTTCG TCACATCTCATCTCATCTACCTC3'; firefly luciferase reverse 5'CCA CATATCAAATATCCGAGTGT3'.

The expression of all genes was normalized to $\beta$-actin.

\section{Aldehyde dehydrogenase activity}

Aldehyde dehydrogenase activity was determined using the ALDEFLOUR staining kit (StemCell Technologies, Grenoble, France) according to the manufacturer's instructions and as described previously. ${ }^{6}$

\section{Statistical analysis}

Statistical analyses were performed using GraphPad Prism software (GraphPad, La Jolla, CA) using paired or unpaired two-tailed Student's $t$-test or ANOVA (analysis of variance), followed by Newman-Keuls post-test. Error bars represent s.d.

SUPPLEMENTARY MATERIAL is linked to the online version of the paper at http://www.nature.com/mi

\section{ACKNOWLEDGMENTS}

We thank Ann-Charlotte Selberg for valuable technical assistance and $\operatorname{Dr}$ A Mowat (Glasgow University) for invaluable comments on this manuscript. This work was supported by grants from the Swedish Medical Research Council, a clinical research grant with the Swedish National Health Service, the Crafoordska, Torsten and Ragnar Söderbergs, Kocks, Österlund and Richard and Ruth Julins Foundations, 
the Royal Physiographic Society, the Wellcome Trust, and the Swedish foundation for Strategic Research FFL-2 program.

\section{DISCLOSURE}

R.B. has an interest in AS Vitas, Oslo, Norway. The remaining authors declare no conflict of interest.

(C) 2011 Society for Mucosal Immunology

\section{REFERENCES}

1. Coombes, J.L. \& Powrie, F. Dendritic cells in intestinal immune regulation. Nat. Rev. 8, 435-446 (2008).

2. Rescigno, M. \& Di Sabatino, A. Dendritic cells in intestinal homeostasis and disease. J. Clin. Invest. 119, 2441-2450 (2009).

3. Johansson-Lindbom, B. et al. Functional specialization of gut CD103+ dendritic cells in the regulation of tissue-selective T cell homing. J. Exp. Med. 202, 1063-1073 (2005).

4. Milling, S., Yrlid, U., Cerovic, V. \& MacPherson, G. Subsets of migrating intestinal dendritic cells. Immunol. Rev. 234, 259-267 (2010).

5. Jaensson, E. et al. Small intestinal CD103+ dendritic cells display unique functional properties that are conserved between mice and humans. J. Exp. Med. 205, 2139-2149 (2008).

6. Schulz, O. et al. Intestinal CD103+, but not CX3CR1+, antigen sampling cells migrate in lymph and serve classical dendritic cell functions. J. Exp. Med. 206, 3101-3114 (2009).

7. Coombes, J.L. et al. A functionally specialized population of mucosal CD103+ DCs induces Foxp3+ regulatory T cells via a TGF-beta and retinoic acid-dependent mechanism. J. Exp. Med. 204, 1757-1764 (2007).

8. Worbs, T. et al. Oral tolerance originates in the intestinal immune system and relies on antigen carriage by dendritic cells. J. Exp. Med. 203, 519527 (2006)

9. Sun, C.M. et al. Small intestine lamina propria dendritic cells promote de novo generation of Foxp3 T reg cells via retinoic acid. J. Exp. Med. 204, 1775-1785 (2007).

10. Iliev, I.D. et al. Human intestinal epithelial cells promote the differentiation of tolerogenic dendritic cells. Gut. 58, 1481-1489 (2009).

11. Kang, S.G., Lim, H.W., Andrisani, O.M., Broxmeyer, H.E. \& Kim, C.H. Vitamin A metabolites induce gut-homing FoxP3+ regulatory $T$ cells. J. Immunol. 179, 3724-3733 (2007)

12. Blomhoff, R. \& Blomhoff, H.K. Overview of retinoid metabolism and function. J. Neurobiol. 66, 606-630 (2006).

13. Guilliams, M. et al. Skin-draining lymph nodes contain dermis-derived CD103(-) dendritic cells that constitutively produce retinoic acid and induce Foxp3(+) regulatory T cells. Blood 115, 1958-1968 (2010).

14. Yokota, A. et al. GM-CSF and IL-4 synergistically trigger dendritic cells to acquire retinoic acid-producing capacity. Int. Immunol. 21, 361-377 (2009).

15. Chang, S.Y. et al. Lack of retinoic acid leads to increased langerinexpressing dendritic cells in gut-associated lymphoid tissues. Gastroenterology 4, 1468-1478 (2009).

16. Persson, E.K., Jaensson, E. \& Agace, W.W. The diverse ontogeny and function of murine small intestinal dendritic cell/macrophage subsets. Immunobiology 215, 692-697 (2010).

17. Agace, W.W. T-cell recruitment to the intestinal mucosa. Trends Immunol. 29, 514-522 (2008).

18. Rescigno, M., Lopatin, U. \& Chieppa, M. Interactions among dendritic cells, macrophages, and epithelial cells in the gut: implications for immune tolerance. Curr. Opin. Immunol. 20, 669-675 (2008).

19. Artis, D. Epithelial-cell recognition of commensal bacteria and maintenance of immune homeostasis in the gut. Nat. Rev. 8, 411-420 (2008).

20. Shale, M. \& Ghosh, S. How intestinal epithelial cells tolerise dendritic cells and its relevance to inflammatory bowel disease. Gut 58, 1291-1299 (2009).

21. Szatmari, I. et al. PPARgamma controls CD1d expression by turning on retinoic acid synthesis in developing human dendritic cells. J. Exp. Med. 203, 2351-2362 (2006)

22. Manicassamy, S. et al. Toll-like receptor 2-dependent induction of vitamin A-metabolizing enzymes in dendritic cells promotes $T$ regulatory responses and inhibits autoimmunity. Nat. Med. 15, 401-409 (2009).

23. Elgueta, R. et al. Imprinting of CCR9 on CD4 T cells requires IL-4 signaling on mesenteric lymph node dendritic cells. J. Immunol. 180, 6501-6507 (2008).

24. Bogunovic, M. et al. Origin of the lamina propria dendritic cell network. Immunity 31, 513-525 (2009).

25. Varol, C. et al. Intestinal lamina propria dendritic cell subsets have different origin and functions. Immunity 31, 502-512 (2009).

26. Iwata, M. et al. Retinoic acid imprints gut-homing specificity on T cells. Immunity 21, 527-538 (2004).

27. Svensson, M. et al. Retinoic acid receptor signaling levels and antigen dose regulate gut homing receptor expression on CD8+ T cells. Mucosal. Immunol. 1, 38-48 (2008).

28. Saurer, L., McCullough, K.C. \& Summerfield, A. In vitro induction of mucosa-type dendritic cells by all-trans retinoic acid. J. Immunol. 179, 3504-3514 (2007).

29. Ong, D.E., Tsai, C.H. \& Chytil, F. Cellular retinol-binding protein and retinoic acid-binding protein in rat testes: effect of retinol depletion. J. Nutr. 106, 204-211 (1976).

30. Naik, S.H. et al. Intrasplenic steady-state dendritic cell precursors that are distinct from monocytes. Nat. Immunol. 7, 663-671 (2006).

31. Naik, S.H. et al. Development of plasmacytoid and conventional dendritic cell subtypes from single precursor cells derived in vitro and in vivo. Nat. Immunol. 8, 1217-1226 (2007).

32. Barker, N. et al. Identification of stem cells in small intestine and colon by marker gene Lgr5. Nature 449, 1003-1007 (2007).

33. Hicks, V.A., Gunning, D.B. \& Olson, J.A. Metabolism, plasma transport and biliary excretion of radioactive vitamin $A$ and its metabolites as a function of liver reserves of vitamin A in the rat. J. Nutr. 114, 1327-1333 (1984).

34. Dunagin, P.E. Jr., Zachman, R.D. \& Olson, J.A. The identification of metabolites of retinal and retinoic acid in rat bile. Biochim. Biophys. Acta. 124, 71-85 (1966).

35. Zachman, R.D., Singer, M.B. \& Olson, J.A. Biliary secretion of metabolites of retinol and of retinoic acid in the guinea pig and chick. J. Nutr. $\mathbf{8 8}$, 137-142 (1966).

36. Nath, K. \& Olson, J.A. Natural occurrence and biological activity of vitamin A derivatives in rat bile. J. Nutr. 93, 461-469 (1967).

37. Feng, T., Cong, Y., Qin, H., Benveniste, E.N. \& Elson, C.O. Generation of mucosal dendritic cells from bone marrow reveals a critical role of retinoic acid. J. Immunol. 10, 5915-5925 (2010).

38. Hammerschmidt, S.I. et al. Stromal mesenteric lymph node cells are essential for the generation of gut-homing T cells in vivo. J. Exp. Med. 205, 2483-2490 (2008).

39. Molenaar, R. et al. Lymph node stromal cells support dendritic cellinduced gut-homing of T cells. J. Immunol. 183, 6395-6402 (2009).

40. Inagaki, T. et al. Regulation of antibacterial defense in the small intestine by the nuclear bile acid receptor. Proc. Natl Acad. Sci. USA 103, 3920-3925 (2006).

41. Vavassori, P., Mencarelli, A., Renga, B., Distrutti, E. \& Fiorucci, S. The bile acid receptor FXR is a modulator of intestinal innate immunity. J. Immunol. 183, 6251-6261 (2009).

42. Pabst, O. et al. Chemokine receptor CCR9 contributes to the localization of plasma cells to the small intestine. J. Exp. Med. 199, 411-416 (2004).

43. Zal, T., Volkmann, A. \& Stockinger, B. Mechanisms of tolerance induction in major histocompatibility complex class II-restricted T cells specific for a blood-borne self-antigen. J. Exp. Med. 180, 2089-2099 (1994).

44. Gundersen, T.E., Bastani, N.E. \& Blomhoff, R. Quantitative highthroughput determination of endogenous retinoids in human plasma using triple-stage liquid chromatography/tandem mass spectrometry. Rapid Commun. Mass Spectrom. 21, 1176-1186 (2007).

\section{(c)}

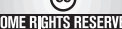

This work is licensed under the Creative Commons Attribution-NonCommercial-No Derivative Works 3.0 Unported License. To view a copy of this license, visit http://creativecommons.org/licenses/by-nc-nd/3.0/ 